\title{
UN ACERCAMIENTO A UNA PERSPECTIVA ECOLÓGICA EN LA EDUCACIÓN
}

\author{
William Delgado Montoya*
}

Este articulo propone un acercamiento a la educación desde la perspectiva ecológica, entendida como la adaptación y ajuste entre un ser humano activo en crecimiento y los escenarios naturales y sociales. En este sentido, plantea la educación como una estructura social que favorece los mecanismos de acomodación al explicar, predecir, interpretar y transmitir la cultura y la práctica social, configurando los escenarios donde se efectúan los procesos de enseñanza- aprendizaje. de esta manera el aula escolar se transforma en un espacio de interacciones entre los individuos (profesores y alumnos). En consecuencia. el enfoque ecológico en la educación es una oportunidad para realizar investigaciones que valoren la animación cultural, el contexto de aprendizaje de los alumnos, las interacciones humanas que constantemente se modifican, para generar una búsqueda de soluciones a los problemas de una manera altruista.

\begin{abstract}
Proposal of an approach to education from an ecological perspective. This perspective is understood as the adaptation and adjustment between a growing and active human being and the natural and social scenarios that surround. Education is seen as a social structure favoring adaptation mechanisms by means of explaining, predicting, interpreting and transmitting social culture and practices, and by configuring the different scenarios where teaching-learning principles take place. Thus, the classroom becomes a place that fosters interactions among individuals (teachers and students). As a result. an ecologic al perspective in edu. cation is an opportunity to develop research that values cultural animation, students' learning context, and the ever changing human interaction in order to promote an altruistic search for solutions to the various problems encountered.
\end{abstract}

* Licenciado en Educación Básica en I y Il Ciclos. Universidad Nacional. Estudiante de la Maestría en Planificación Curricular. Universidad de Costa Rica. Profesor en Ejercicio en la Escuela Ricardo Jiménez. 
Compartir conceptos es esencial para la comunicación de ideas, puntos, problemas y soluciones dentro de cualquier temática, en este caso, a través de un ensayo trato la ecología y la educación, ofreciendo un marco referencial para una discusión coherente de una amplia y compleja relación.

La ecología encuentra sus orígenes en el pensamiento de Darwin, señala Nagi (1984), que, este poseía un concepto muy agudo de las relaciones infinitamente complejas de una especie con otros seres orgánicos. Su concepto deriva del griego "oikos" que significa "casa".

Los humanos como especie han desarrollado complejas culturas, como formas de organización social, debido a la naturaleza y la capacidad de comprender y comunicarse simbólicamente, visualizado en valores, normas y estructuras sociales. Dentro de estos "ecosistemas", comprendidos como estructuras sociales, citó primeramente la familia como la organización social que busca el ajuste del ser humano en el medio ambiente, siendo la unidad o "casa", que desempeña las funciones socioculturales que para la autora ejemplifica: la reproducción, protección, apoyo, alimentación, socialización y transmisión de la cultura, entre otras.

La familia o el hogar es vital para la adaptación del sujeto en su medio, conforman parte del "hábitat" que favorece en la asimilación como una especie, en la biodiversidad de "ecosistemas" y, facilita las condiciones ambientales necesarias, que constituye un "nicho humano", determinando la ocupación y convivencia del individuo para un estilo de vida en sociedad. Con el tiempo, crecen las unidades sociales de familias y tribus a comunidades y sociedades más complejas, en dicha expansión se originan nuevos mecanismos de acomodación y se auspicia el surgimiento de otras formas de organización social, que por las tendencias de los sistemas de valores, orientados en las relaciones con el ambiente natural, con otra especies y otros seres humanos. Se designan a numerosas funciones a favor de estos organismos sociales especializados como es el caso de la educación, la salud y el mercado que resalta el vínculo entre el individuo y su medio sociocultural.

La autora opina que la mayoría de las sociedades contemporáneas han evolucionado como instituciones sociales organizadas alrededor de valores importantes, que constituyen el papel fundamental de las entidades como la educación e información. Es decir, una sincronización, en el sentido de que facilita los procesos vitales, donde prioriza que el individuo se acople a ciertos aspectos del medio e intenta, al mismo tiempo controlarlos, como se ha observado a través de la historia humana. Esta perspectiva ecológica, adquiere muchas implicaciones, al percibir al sujeto como una persona que se reorganiza a lo largo del tiempo y el espacio por diferentes aspectos provenientes del entorno. 
El medio ambiente es un concepto flexible que puede referir al hábitat inmediato, a las sociedades, naciones, regiones del mundo y el mundo mismo, aquí esas condiciones incluyen la composición social en que el ser humano está inmerso. Por eso, al hablar de ecología, discutimos sobre esferas socioculturales que involucran a la humanidad y la humanización del medio. Por ejemplo, dentro de la interdependencia de los elementos en un ecosistema, origina la necesidad de que se promuevan relaciones entre los seres y su medio, poniendo desde luego, énfasis en la socialización temprana, que es a través de la educación, buscando el respeto y cuidado de las condiciones ecológicas en la formación del niño y niña de manera integral.

A pesar de que no se puede analizar posiblemente una sociedad como un sistema cerrado, como principio de la visión de un ecosistema, la educación es en esencia un sistema, donde se proporcionan servicios a los sujetos que deben adaptarse. Es decir, se aborda un enfoque ecológico del desarrollo único experimentado por cada ser humano que al mismo tiempo concibe al sujeto y su relación dinámica, como parte inseparable de los escenarios en los que funciona durante toda su vida. Según la ecología del desarrollo humano, propuesta por Bronfenbrenner (1979) y la relación con el entorno planteada por Thomas y Marshall (1977) que fundamenta la "concepción evolutiva del ambiente ecológico de la persona y su relación con éste" y, la creciente capacidad de la persona para "descubrir, sostener o alterar sus prioridades"; no obstante, apuntan que la relación de ajuste o desarrollo continuo va de la mano con la función de la educación.

Sin lugar a dudas, dentro de la perspectiva ecológica, la posición epistemológica de "educación", citada por Grundy (1994) como Ciencias de la Educación, es acertada por que:

Explica, predice, interpreta y transmite la cultura y la práctica social como un fenómeno interaccional transformador. (p. 143).

En este sentido, educar implica por lo tanto, transformar la sociedad, de allí que sea complejo, dinámico y comprometido. Es complejo debido a que su insumo son los seres humanos. Dinámico y comprometido porque como hecho sociohistórico se ubica en circunstancias y contextos sociales particulares a los que pretende y debe dar respuestas (Carr y Kemmis, 1988).

De alguna manera, la relación entre ecología y educación se puede comprender en el marco de un contexto, refiere García, V.. Álvarez, S., Guerrero A., Desantes, J., Fernández, O., Losado, I., García, J., Marín, C., Martínez, A., Pérez, R. y Soler, E. (1996) que el contexto es un "conjunto de elementos físicos, espaciales, temporales, sociales y culturales" que configuran los escenarios en que tienen lugar los procesos de enseñanza-aprendizaje. 
Una corriente pedagógica ecologística, en la cuál, orienta al ser humano, el descubrir la naturaleza de su entorno apropiado para su desarrollo potencial como máxima expresión del orden natural que debe construir sin destruir el entorno físico, natural y social.
Igualmente sostiene Frederick Erickson y Jeffrey Shultz (1981) quienes apuntan que contexto es " lo que la gente hace, dónde, cuándo y cómo lo hacen".

Hamilton, citado por Wittorck (1997) conceptualiza el aula escolar o salón de clase, como "medios comunicativos, en los cuales los hechos que constituyen la vida cotidiana se entienden como parte de las interacciones entre profesores y estudiantes". Es decir, el salón de clase son las interacciones entre los individuos y su convivencia entre sí.

Por otro lado, considero importante referirme al término "relación" que comprende la correspondencia y conexión entre un objeto con otro. En este sentido, dentro del ensayo se establece el término "relación", como la conexión o correspondencia existente entre una perspectiva ecológica y la educación. como por ejemplo, dentro del contexto escolar, específicamente el aula se observa la relación entre educando y educador.

Para explicar un poco más estas relaciones, durante las décadas de 1940 y 1950, el énfasis en el campo educativo se centraba en las causas biológicas y en intervenciones por medio de la rutina. la realización de ejercicios, la instrucción secuencial y la redacción de estímulos ambientales.

En la década de 1960, Jones (1986) se refiere a cómo en la perspectiva ecológica dentro de la educación, predomina la intervención psicológica y las relaciones interpersonales, actualmente está centrada en las intervenciones individualizadas, en inferir las causas de la conducta inapropiada y en el mejoramiento de las relaciones interpersonales. Después de este período existió un énfasis en el uso de las intervenciones conductuales, que se enfocaban en los principios del aprendizaje y en el control o modificación del comportamiento del estudiante, enfatizando las habilidades de organización y manejo del salón de clase de manera simple, a menudo superficial, orientado al control.

Con la aplicación de la perspectiva ecológica, se realiza un cambio desde un enfoque que integra una perspectiva biofísica, conductual, psicológica y educativa dentro de las estructuras de los sistemas, como reitera Jones (1986).

En esta perspectiva. se aplican los principios del aprendizaje (conductual) con consideraciones a las relaciones interpersonales (psicológica y educativa) y con reconocimiento del impacto de los factores neurológicos y físicos (biofísica) al funcionamiento de los estudiantes, que algunos lo definen como "aprendices" 
en el aula o salón de clases. Esta visión, tiene implicaciones significativas para el manejo del aula o salón de clase, en particular con respecto a la interacción entre el docente y el alumnado. Sugiere Bronfernbrenner (1979) que el aprendizaje y el desarrollo se facilita cuando la persona en crecimiento, participa en patrones progresivamente más complejos de actividad recíproca, con alguien con quien ha establecido una relación personal fuerte y perdurable.

El aprendizaje ocurre mediante la posibilidad de un cambio gradual en la persona en formación. Los docentes, entonces, deben relacionarse con los estudiantes como personas capaces, individuos aptos para hacer elecciones y manejar su propio comportamiento. Destacando la interacción entre el escenario, el estudiante, el educador y todos los otros agentes externos que "actúan" dentro del contexto, como padres de familia, personal administrativo, entre otros (Bauer y Shea, 1999).

De acuerdo con Green y Weade (1998), lo que ocurre en el aula, con respecto a la construcción del conocimiento, por ejemplo, sucede durante la interacción con otros, donde la comunicación que se suscita en el escenario del aula escolar afecta: a) qué estudiante tiene oportunidad de aprender; b) qué aprende en realidad; y c) cuál alumno tiene la oportunidad de exhibir su conocimiento y aprendizaje.

En consecuencia, el enfoque ecológico en la educación, ofrece la oportunidad de realizar investigaciones que consideren el aprendizaje de los alumnos y sus habilidades para la solución de problemas de interacciones como una medida de resultados central en la determinación entre el papel y funcionalidad del docente y el aula, como se plantea en las propuestas teóricas, como por ejemplo de Piaget, Vigosky y Brunner. Además en corrientes pedagógicas. en la investigación acción y en la investigación protagonista, que ubica el currículo como una de las teorías funcionalistas y el objetivo de la educación es el cambio cultural y colectivo (Carvajal, 1998).

En este referencial, existen dos tendencias curriculares: el currículo emergente y el integrador, como señala Carvajal (1998). El currículo integrador es la organización de los contenidos a partir de temas, problemas o proyectos concretos que usualmente surgen del análisis de las necesidades locales y en que se relacionan diversas disciplinas, incluyendo actividades de servicio y producción. Claramente, son hermenéuticos por que el conocimiento resulta de una elaboración de la realidad, lo que promueve a su vez su transformación, así como esta concepción se fundamenta en su posición fenomenologista, donde lo más importante es la causa de estos y las afinidades entre elementos que los unen entre sí.

El autor considera que la función social de la educación es crear condiciones para la armonía de los grupos, buscando al mismo tiempo que se vayan liberando de sus limitaciones, o sea crea cultura, desarrolla el diálogo y la 
conciencia colectiva, frente a un espíritu crítico. Esta posición, al mismo tiempo es sustentada por una corriente pedagógica ecologística, en la cuál, orienta al ser humano, el descubrir la naturaleza de su entorno apropiado para su desarrollo potencial como máxima expresión del orden natural que debe construir sin destruir el entorno físico, natural y social.

No obstante, se busca además, una armonía colectiva, una igualdad de derechos en relación con los recursos y el desarrollo individual y colectivo. De esta manera, la educación debe promover el desarrollo armónico y equitativo de la humanidad del cual todos son responsables, como postula Powell, Maturana y diversos movimientos ecologistas en los últimos años (Carvajal, 1998).

La perspectiva ecológica para Copeland (1982), sostiene que las afinidades en las aulas, se asumen sus características conforme a los docentes y los alumnos que influyéndose recíprocamente, desde aquí nace la pertinencia de promover un espacio adecuado para el crecimiento armonioso y equitativo. Pinnell y Galloway (1987) resumen el enfoque del desarrollo del manejo del aula escolar, donde los docentes reconocen que los educandos contribuyen en forma significativa al proceso educativo, así como el aprendizaje ocurre cuando los estudiantes sienten la necesidad de cambiar o aprender; además, el aprendizaje es holístico, en lugar de una serie de partes de información o habilidades individuales, estas reconocen el poder del contexto social de la escuela en el aprendizaje, integrando una comprensión personal del aprendizaje y el avance, preocupándose por lo que ocurre en nuestras aulas. Aunque como es de nuestro conocimiento, no es el único escenario, el salón de clase o aula escolar, puede servir como una "vitrina" para identificar las debilidades del entorno donde funcionan o se ven inmersos los alumnos. Es decir, los educadores pueden "diagnosticar" el lugar en que convive el niño o la niña, permitiendo al mismo tiempo, buscar soluciones al contexto inmediato en que se ven involucrados los individuos, tomándose en cuenta la forma dinámica en que están implicados los seres humanos en la formación del educando.

Desde la perspectiva ecológica, el desarrollo humano es la acomodación mutua y progresiva, o adaptación y ajuste, entre un ser humano activo en crecimientos y los escenarios y contextos ecológicos más amplios en los que se insertan (Bauer y Shea, 1999). Por lo cuál, el comprender "los escenarios" en que interactúan nuestros alumnos permitirían un aprendizaje atinente a sus intereses y necesidades.

Delors (1996) opina que los seres humanos están más concientes de las amenazas que pesan sobre su medio ambiente, tanto humano como natural, pero todavía no se ha dotado de los medios para remediar esa situación, a pesar de las diversas reuniones internacionales, citando por ejemplo, como desde 1945 la 
humanidad ha vivido 150 guerras que han causado 20 millones de muertes, antes y también después de la caída del muro de Berlín. Esto genera tensión entre lo mundial y lo local, lo universal y lo singular, promoviendo nuevas perspectivas como es el caso del enfoque ecológico, que se plasma en la filosofía de una "aldea planetaria", como respuesta o posibles soluciones rápidas, que enseñan a vivir mejor mediante el conocimiento, la experimentación y la formación de una cultura personal, generando nuevas relaciones entre nación, grupo e individuo.

Frente a los numerosos desafíos, la relevancia social de la educación constituye, según Delors (1996), un "instrumento indispensable" para que la humanidad pueda progresar hacia los ideales de paz, libertad y justicia social en el desarrollo continuo de la persona y la sociedad, el hacer retroceder la pobreza, la exclusión, las incomprensiones, como las guerras que perjudican los contextos ecológicos del desarrollo humano.

Al mismo tiempo la educación forma parte de un clamor por la infancia y la juventud, donde cada ecosistema en los contextos ecológicos humanos y naturales, fortalecen el crecimiento humano que refleja una visión de una instancia educativa, siendo elemental para crear espacios de estabilidad, de recursos humanos y ambientales. Por eso, plantea que mediante las nuevas formas de actividad económica, social y, mediante lo que denomina "los cuatro pilares de la educación: aprender a vivir juntos, aprender a conocer, aprender a hacer y aprender a ser", que dotan al sujeto una competencia para adaptarse al entorno, ya que para Thomas y Marshall (1977):

“...rara vez se adopta, nunca lo hace por completo, a las necesidades específicas de un individuo frente a su medio. Por consiguiente, el propósito último de cualquier programa de educación es el asistır a este sujeto para que se adopte lo mejor posible a su entorno" ( p. 16).

Así, se justifica la importancia de que el ser humano, aprenda a ser un individuo que esté en constante formación, o sea que durante toda la vida, se faculte con las herramientas esenciales para el aprendizaje y sincronización del desarrollo pleno de sus capacidades. El logro de una participación activa de la comunidad, debe partirse de una evaluación de las necesidades, mediante el diálogo con las autoridades públicas y los grupos interesados dentro de la sociedad, siendo una primera etapa fundamental, para ampliar el acceso a la educación y mejorarla, considerándolos de simples "protagonistas escuchas" a sujetos más autónomos, emancipadores y críticos, con una visión democrática, global, dialéctica de la realidad emergente (De Alba, 1994; Delors, 1996; Torres, 1996).

Según Bronfernbrenner (1979), los contextos ecológicos o escenarios, muestran el desarrollo de un individuo que anida, uno dentro del otro, como 
parte de su esencia espiritual, emocional y física, citando el ejemplo de un conjunto de muñecas rusas, específicamente a las relaciones interpersonales, coherentes escenarios, afín entre las interacciones de grupo, la sociedad y el individuo. Ante esto, cualquier cambio individual debe comprenderse dentro del contexto del sistema social y cultural más amplio (Riegel, 1975).

Desde una perspectiva de la educación, los escenarios específicos de mayor relevancia inicial para el crecimiento del sujeto son la escuela, la familia, el vencindario y la comunidad a la que pertenece, concibiéndolos personas dinámicas y en progreso.

La dimensión ecológica como parte de un estilo pedagógico se manifiesta en un modelo curricular, donde el proceso de enseñanza-aprendizaje, se produce en un ecosistema, el cual comparte una dinámica y una serie de interdependencias en lo académico. Para Ander- Egg (1996), dentro del pensamiento psicopedagógico con el nombre de "ecología escolar", considera los factores del entorno físico y psicosocial como condicionantes de la conducta individual y grupal.

A partir de esta inserción en un marco de relaciones e interrelaciones globales, se han fomentado, alertado y creado formas de acción. para que el alumno se comprometa en la transformación de la sociedad. En el ambiente local, de aprendizaje y el clima de las clases se consideran los factores como ambiente físico, los comportamientos y actitudes personales, la organización que refleja la dinámica interna del grupo humano de clase, el intercambio comunicativo y la relación socioafectiva que existe en un grupo humano, en este caso, dentro de un aula escolar, visualizado en el marco constituido por los docentes y alumnos.

Ander- Egg (1996) ejemplifica el fenómeno multidimensional que se observa en el acto del conocimiento en un contexto escolar de la siguiente manera:

Acto del conocimiento

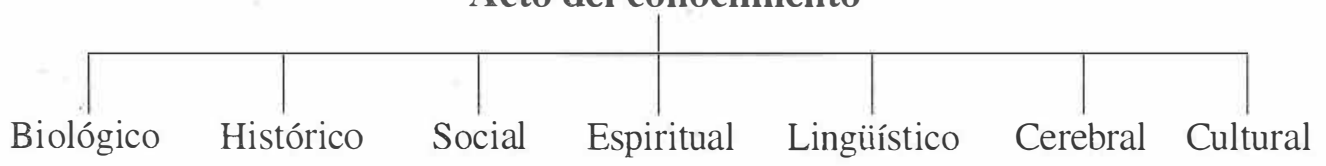

Peralta (1987) comenta que los factores y elementos que componen todo fundamento ecológico del currículo inicial es:

“...la relación y preservación del entorno natural es un aspecto que ha sido detectado como prioritario en nuestra sociedad actual...de una base ecológica, que junto con favorecer un mejor conocimiento de ese medio, favorezca en especial el respeto hacia la naturaleza" (p. 22). 
Por esta razón, la relación y preservación del entorno natural colabora en el aprendizaje significativo del educando. Pérez (1994) cita cómo la estructura de las grandes sociedades en sus órdenes tanto político, económico y social, exigen del ser humano el despliegue de numerosas estrategias y capacidades para comprender, interpretar y comprometerse en el destino, tanto individual como colectivo en un proceso interactivo.

En este sentido, que se concreta en una pedagogía de una cultura, ciencia y arte; visualizada como una "animación cultural" que es determinada por un contexto institucional singular, donde las relaciones e intercambios humanos se modifican, tanto en las interacciones verbales como no verbales que conforman el contexto ecológico del salón de clases y la escuela en general.

Hamilton, citado por Wittorck (1997), afirma cómo dentro del contexto escolar, específicamente el aula, se intenta comprender la naturaleza de las interacciones en los procesos de enseñanza-aprendizaje, que conforma la investigación ecológica en cuatro criterios: a) atención a la interacción recíproca entre las personas, b) las consideraciones de la enseñanza y el aprendizaje como procesos continuamente interactivos, c) el contexto como la escuela, la comunidad, la familia y la cultura, d) las fuentes importantes de datos a los procesos no observados, tales como los pensamientos, actitudes, sentimientos o percepciones de los participantes.

Paulo Freire (1988) apunta que la educación es una "praxis, reflexión y acción del ser humano sobre el medio para transformarlo", explicando que de alguna manera para influir en la realidad es indispensable encontrarnos inmersos en una acción que posibilite la transformación y la mejora de la misma, lo que exponía Pérez como una "animación sociocultural".

Esta animación cultural constituye el desarrollo como un proceso integral, que incluye los cambios, procesos y mecanismos que impulsan los avances que todos y cada una de los seres humanos se dan a lugar, desde el momento de la concepción del individuo, que como expresa León (1998), el aprendizaje lleva tres áreas que son: el desarrollo físico, el desarrollo socioemocional y el cognitivo.

La autora cita que dentro del desarrollo físico, el bagaje genético es característico ante un crecimiento como un estado fisiológico manifestado por la motora gruesa y fina y, la maduración en el sujeto. En el área del desarrollo cognitivo lingüístico, retoma la idea del bảgaje genético en dos subáreas que son la nutrición, como el medio familiar que estimula y atiende al niño o niña, facultando de las habilidades necesarias para la asimilación del medio. También se expone, la subárea de la maduración, que explica cómo el individuo se enfrente al medio escolar, ante los procesos de enseñanza-aprendizaje que ajustan las relaciones con el grupo etáreo y de los profesores. 
Finalmente, en el área socioemocional entra el carácter cultural y medio social comunitario, junto con la familia en que se dan relaciones con otros sujetos, estimulando el autoconcepto y la autoestima propia del niño y la niña.

De esta forma, la construcción progresiva del desarrollo humano en el enfoque ecológico es la adquisición de habilidades, destrezas y conocimientos del proceso de transformación de su entorno, como lo muestra Bauer y Shea (1999) en el siguiente cuadro:

\begin{tabular}{|l|l|}
\hline SOCIEDAD & valores y culturas \\
\hline Interacciones grupales & escenarios, vecindario, trabajo \\
\hline Relaciones entre escuela & padres/ maestros, escuela/ hogar \\
\hline Relaciones interpersonales & $\begin{array}{l}\text { Impacto en las interacciones familiares } \\
\text { yel salón de clase, su identificación y } \\
\text { valoración. }\end{array}$ \\
\hline INDIVIDUO & $\begin{array}{l}\text { En el ámbito cognitivo, psicomotory } \\
\text { fisico (aprendizaje, lenguaje y } \\
\text { comunicación social y emocional). }\end{array}$ \\
\hline
\end{tabular}

El modo de pensamiento integrativo que ofrece la perspectiva ecológica. sustenta implicaciones que no solo son importantes para la educación, la ciencia y filosofía, sino que también para las diversas estructuras sociales, políticas e inclusive económicas.

En este caso, las actividades económicas se involucran en el enfoque ecológico, proporcionando una urgente perspectiva a los economistas, como señala Capra (1994):

es un sistema vivo compuesto por seres humanos y organizaciones sociales en continua interacción con los ecosistemas ambientales de los que dependemos, siendo su propósito el favorecer el desarrollo, o sea aquella consecución del máximo de producción y consumo para el bienestar humano tanto en su salud y suplencia de necesidades materiales, emocionales y espirituales que intentan el equilibrio social y ecológico (p. 31). 
Se pretende lograr un concepto cualitativo del desarrollo económico, que al mismo tiempo, busca un cambio en los nuevos valores, junto con nuevas actitudes y estilos de vida. Siendo su aplicación en los procesos y actividades sociales, culturales, económicas y políticas prácticamente favorable para una perspectiva: en la cual, el bienestar humano es la meta, sin dejar de lado la preservación ambiental.

Igualmente es bastante obvio que en el naciente siglo XXI encontramos grandes retos, que dependerán de una visión y capacidad adecuada para enfrentarlos y que, en consecuencia, el horizonte para las futuras generaciones sea apto o no. De esta manera, no caemos en el error que nos previene Goleman (1998) sobre la analfabetización "emocional" existente, que para este ensayo la podríamos comprender como analfabetización "ecológica educativa", en cuanto perdemos de vista al estudiante como un ser integral y que es mutado por los organismos sociales en que se ve sumergido, como es la educación.

Sartre decía que el ser humano es esclavo de la libertad, siendo condenado a ejercerla y es donde nuestras decisiones favorecen o perjudican una mejor calidad de vida a los futuros individuos sociales. Por lo cual, la dimensión ecológica en la educación, fundamentada por movimientos desde los principios de los ochentas como son los movimientos ecologistas, pacifistas y feministas, el conocimiento de la salud holística y el potencial humano, distintas corrientes espirituales y numerosas iniciativas de ciudades, como movimientos a favor del Tercer Mundo o de la liberación étnica, han originado una coalición para la transformación oportuna de nuestra sociedad actual, ejemplo de esta unión es el Movimiento Verde Europeo (Capra, 1994).

La escuela por tanto, debe ofrecer los medios necesarios para la acomodación, asimilación y adaptación del sujeto en su medio. Ya que, en la educación debe partir de la naturaleza reiterativa que fortalece el desarrollo del individuo. En palabras del padre Thomas Berry, citado por Gore (1993):

el universo es una comunicación de sujetos y no un complejo de objetos (p. 197).

El enfoque ecológico en el contexto educativo nos ofrece el idioma indispensable para la comunicación entre los sujetos y su medio y no verlos con objetos que lo conforman. En este sentido, la perspectiva ecológica permite a los individuos comprender más su funcionalidad y priorizar al mismo tiempo qué aprendizajes delega en las personas.

Por tanto, en las instituciones debe existir una reorientación bajo este enfoque en donde se brinde mayor participación estudiantil y contacto con el sector 
productivo y de servicios, así como la permeabilidad ante la realidad social y una actividad educativa originada en los intereses de la comunidad local o inmediata.

En este sentido, se establece la relación entre el docente y el educando, facilitándose la expresión y estimulándose la creatividad, por que se considera a cada persona única y diferente de las demás, pero comprometida con sus iguales y siempre en la búsqueda de soluciones a los problemas de una manera altruista. Sin lugar a dudas. la educación deberá por tanto centrarse en cada ser humano y al mismo tiempo en los escenarios en que convive, promoviendo una persona activa, emancipatoria y gestora de su aprendizaje, sobresaliendo el respeto por sí mismo como protagonista, apropiándose de su cultura, en su medio ambiente humano y natural.

La perspectiva ecológica en la educación enriquece, no sólo la adquisición de conocimiento en el alumnado, sino también las adaptaciones que promueven un equilibrio integral entre hábitat y especie. Es decir, facultad al estudiante para "acogerse" dentro de un medio natural y social, donde el papel del educador es relevante, como bien lo dice Joaquín García Monge en un fragmento del discurso pronunciado en 1917, con motivo de la Tercera graduación de la Escuela Normal:

"El educador no es un domador, es un modelador. No es un verdugo, sino un profeta. No es un empleo que gana sueldos para comer, sino un aislado que vela en silencio por el bien de los demás.

No es un miserable, es un poderoso; juega con el infinito porque trabaja con el alma.

El maestro no compone, demuele y transforma. Demuele principios tóxicos y absurdos, sistemas nocivos y creencias falsas. Reforma temperamentos enfermos, organismos raquíticos, instituciones torcidas y construye sociedades sanas, justas y fuertes".

\section{Referencias}

Ander-Egg, E. (1996). La planificación educativa: conceptos, métodos, estrategias y técnicas para educadores. Buenos Aires, Argentina: Magisterio del Río de La Plata.

Bauer, A. y Shea, T. (1999). Educación especial: un enfoque ecológico. 2 da ed. Nueva York, E.E.U.U.: Mac Graw Hill.

Bronfernbrenner, U. (1979). An integrative perspective on children's divorce adjustment. American psychologist, 36. 856- 866. 
Capra, F. (1994) La red de los problemas que hay en el mundo. Nueva Conciencia, 22. $26-31$.

Carvajal, C. (1998). Teorías educativas, concepciones curriculares y corrientes pedagógicas. Mimeografiado, Escuela de Formación Docente, Facultad de Educación, Universidad de Costa Rica.

Carr, W. y Kemmis, S. (1988). Teoría crítica de la enseñanza. La investigación acción en el profesorado. Barcelona, España: Martínez Roca.

Copeland, C. (1982). Teaching-learning behaviors and the demands of the classroom environment. En W. Doyle y T. Good (Eds). Focus in Teaching, 1. 83-97.

De Alba, A. (1994). Currículo, crisis, mito y perspectiva. México: Universidad Nacional Autónoma de México.

Delors, J. (1996). La educación encierra un tesoro. Madrid, España: Editorial Santillana- UNESCO.

Erickson, F. y Schultz, J. (1981). When is context? Some issues and methods in the analysis of social competence, p.p. 147-160. En John Green y Chareles Wallat (Eds.) Ethnography and language in educational settings. Noawood, New Jersey: Editorial Publishing Ablex.

Freire, P. (1998). La educación como práctica de la libertad. Madrid, España: Siglo XXI.

García, J. (1917). Discurso con motivo de la Tercera graduación de la Escuela Normal. Mimeografiado, División de Educación Básica, Centro de Investigación y Docencia en Educación, Universidad Nacional.

García, V., Álvarez, S., Guerrero A., Desantes, J., Fernández, O., Losado, I., García, J., Marín, C., Martínez, A., Pérez, R. y Soler, E. (1996). Formación de profesores para la educación personalizada. Madrid, España: Rialp.

Goleman, D. (1998). La inteligencia emocional. Buenos Aires, Argentina: Javier Vergara.

Gore, A. (1993). La Tierra en juego. Buenos Aires, Argentina: Javier Vergara. 
Green, J. Y Weade, R. (1988 abril). Teaching as conversation and the construction of meaning in the classroom. Ponencia presentada en la reunión Anual de la American Educational Research Association.

Grundy, S. (1994). Producto o praxis del currículo. 7a. ed. Madrid, España: Morata.

Jones, V. (1986). Classroom management in the United States: Trends and critical issues. En D. P. Tattam (eds). Management of disruptive pupil behavior in schools. Chichester, Inglaterra: John Wiley.

Kurdek, L. (1981). An integrative perspective on children's divorce adjustment. American Psychologist, 36. 856- 866.

León, A. (1998). El maestro y los niños: la humanización del aula. San José: Universidad de Costa Rica.

Nagi, S. (1984 mayo). Valores sociales e instituciones y el lugar que ocupa los niños: una perspectiva ecológica. Ponencia presentada en XVI Congreso Panamericano del Niño, Washington D. C., E.E.U.U.

Pinnell, G. y Galloway, C. (1987). Human development, language on communication: Then and now. Theory into practice, 26. 353- 357.

Peralta, V. (1987). El currículo en el jardín infantil: análisis crítico. Mimeografiado, Facultad de Educación, Universidad de Costa Rica.

Pérez, R. (1994). El currículo y sus componentes. Barcelona, España: Oikostar.

Riegel, K. (1975). Toward a dialectical theory of development. Human Development, $18.65-79$.

Savater, F. (1997). El valor de educar. Barcelona; España: Ariel.

Thomas, E. y Marshall, M. (1977). Clinical evaluation and coordination of services: an ecological model. Exceptional Children, 44. 16-22. 
Torres, J. (1996). Globalización e interdisciplinariedad: el currículo integrado. Madrid, España: Morata.

Wittrock, M. (1997). La investigación de la enseñanza: enfoque, teorías y métodos. Barcelona, España: Paidos. 